\title{
Comparison of experimental mouse models of inflammatory bowel disease
}

\author{
SOO YOUN OH ${ }^{1}$, KYUNG-AH CHO ${ }^{2}$, JHHEE LEE KANG ${ }^{3,4}$, KWANG HO KIM ${ }^{1}$ and SO-YOUN WOO ${ }^{2}$ \\ Departments of ${ }^{1}$ Surgery, ${ }^{2}$ Microbiology, ${ }^{3}$ Physiology and ${ }^{4}$ Tissue Injury Defense Research Center, \\ School of Medicine, Ewha Womans University, Seoul 158-710, Republic of Korea
}

Received August 28, 2013; Accepted November 22, 2013

DOI: $10.3892 /$ ijmm.2013.1569

\begin{abstract}
Inflammatory bowel disease (IBD) is multifactorial and involves immunological, environmental and genetic factors. Although there are no animal models that effectively mimic human IBD, experimental models allow us to analyze the mechanisms of chronic intestinal inflammation. IBD can be induced in mice by dextran sulfate sodium (DSS) or by a 2,4,6-trinitrobenzene sulfonic acid (TNBS)-ethanol enema, which evoke immune responses and colitis. In this study, in order to compare the mechanisms of inflammatory response in mice, 3 distinct models of IBD were established: $2 \%$ TNBSinduced acute colitis, 4\% DSS-induced acute colitis and 2\% DSS-induced chronic colitis. In addition, to evaluate the effects of TNBS on inflammasome activation, we used caspase-1 knockout $(\mathrm{KO})$ mice. Changes in both body weight and survival became prominent after day 1 in the $2 \%$ TNBS-induced colitis model, and after day 5 in the 4\% DSS-induced colitis model. The TNBS- and DSS-treated mice, but not the caspase-1 KO mice, showed a massive bowel edema and disruption of epithelial cells. The level of $\mathrm{CD} 11 \mathrm{~b}^{+} \mathrm{Gr}-1^{+}$myeloid-derived suppressor cells (MDSCs) was increased in all tested tissues of the TNBS- and DSS-treated groups, apart from the basal membrane (BM) in the DSS-induced colitis groups and the lamina propria (LP) in the DSS-induced chronic colitis group. We further analyzed different subsets of $\mathrm{CD}^{+} \mathrm{T}$ cells in LP and found that the levels of interferon (IFN) $\gamma$-secreting $\left(\mathrm{IFN} \gamma^{+}\right.$), IL-17-secreting (IL-17 $7^{+}$, but not those of IL-4-secreting (IL-4 ${ }^{+}$) $\mathrm{T}$ cells increased upon treatment with TNBS or DSS. In addition, discrepancies between the histopathologies of wild-type and caspase- $1 \mathrm{KO}$ mice indicated that the pathogenesis of IBD may be associated with the inflammasome pathway responses mediated by caspase-1 in TNBS-induced colitis.
\end{abstract}

Correspondence to: Professor So-Youn Woo, Department of Microbiology, School of Medicine, Ewha Womans University, 911-1 Mok-Dong, Yang-Chun-Gu, Seoul 158-710, Republic of Korea E-mail: soyounwoo@ewha.ac.kr

Key words: inflammatory bowel disease, caspase-1, dextran sulfate sodium, 2,4,6-trinitrobenzene sulfonic acid

\section{Introduction}

Inflammatory bowel disease (IBD) is multifactorial and involves immunological, environmental and genetic factors. Although there are no animal models that effectively mimic human IBD, experimental models allow us to analyze the mechanisms of chronic intestinal inflammation. Animal models of IBD involve genetic manipulation, i.e., either the insertion (transgenic) or selective deletion (knockout) of a gene. In addition, immune responses evoked in animal models are often used as models of IBD for the development and evaluation of drug candidates. For example, 2,4,6-trinitrobenzene sulfonic acid (TNBS)-ethanol enema-induced colitis is associated with chemically induced damage and $\mathrm{T}$ cell immune reactivity. The administration of the sensitizing agent, TNBS, in a $45-50 \%$ ethanol enema induces colitis in rodents. In the TNBS model, ethanol breaks the mucosal barrier and is a crucial component; colitis is not induced by TNBS alone $(1,2)$. In mice, TNBS-induced colitis shows the common delayed hypersensitivity response, mediated by $T$ cells that are sensitized by 'hapten-modified self-antigens'. These self-antigens can be produced by the covalent binding of the hapten trinitrophenyl (TNP) to self-peptides. Tissues from mice with TNBS-induced colitis show an infiltration of $\mathrm{CD}^{+} \mathrm{T}$ helper (Th) cells, mainly Th1 and Th17 (3), and IgG- or IgA-producing B cells (4) in the mucosa and submucosa.

Colitis can be also induced in hamsters, rats and mice by the addition of 30-35 kDa dextran sulfate sodium (DSS) to drinking water at $3-10 \%$ (5). It results in bloody diarrhea, weight loss, shortening of the colon, mucosal ulceration and neutrophilic infiltration. This is a fairly simple and reproducible method used to induce colitis in mice, and DSS-induced colitis is popular for the screening of potential therapeutic agents, with a high number of agents having shown beneficial effects in this model, indicating that it is a sensitive screening model for IBD. However, limitations of this model include the fact that DSS-induced colitis does not require $\mathrm{T}$ or $\mathrm{B}$ cell responses (6), and that luminal bacteria may play a role in the development of this type of colitis (7).

Inflammasomes sense damage signals during infection (e.g., a norovirus infection) and activate caspase-1, leading to the activation of interleukin (IL)-1 $\beta$ and IL-18 by cleaving their pro-forms. Increased levels of pro-inflammatory cytokines, including IL-1 $\beta$, IL- 6 , IL-18 and tumor necrosis factor- $\alpha$ (TNF- $\alpha$ ), have been reported in IBD and correlate with the 
severity of inflammation $(8,9)$. Indeed, enhanced levels of IL-1 $\beta$ have been found in the colonic mucosa and in peritoneal macrophages in the mouse model of colitis and may hence represent an initial trigger of intestinal inflammation (10). In the DSS-induced colitis model, it has been reported that DSS triggers inflammation via the activation of the NLRP3 inflammasome, leading to caspase-1 activation (11), and also via the NLRP6 inflammasome $(12,13)$. In addition, studies using caspase-1 knockout (KO) mice have suggested that caspase-1 contributes to DSS-induced colitis (14).

Myeloid-derived suppressor cells (MDSCs) are a heterogeneous group of pathologically activated myeloid $\mathrm{CD} 11 \mathrm{~b}^{+} \mathrm{Gr}-1^{+}$ cells, consisting of granulocytic and monocytic subsets. MDSCs are recruited from the blood to the site of chronic inflammation, cancer, or transplantation, and modulate innate and adaptive immune responses (15). In the mouse model of colitis, the transplantation of splenic $\mathrm{CD} 11 \mathrm{~b}^{+} \mathrm{Gr}-1^{+}$cells into mice with DSS-induced colitis improved disease parameters (16).

In this study, we compared mouse models of TNBS- and DSS-induced, as well as acute and chronic DSS-induced colitis in terms of histological parameters and profiles of MDSCs and $\mathrm{CD}^{+} \mathrm{T}$ cells. We also investigated the role of caspase- 1 in TNBS-induced colitis using caspase-1 KO mice.

\section{Materials and methods}

Mice. Female, 8-week-old C57BL/6 mice were acquired from Daehan Biolink Co. Ltd. (Samseong-myeon, Korea) and caspase-1 KO mice were generously provided by $\mathrm{Dr}$ F.S. Sutterwala from the University of Iowa (Ames, IA, USA). Mice were housed in an animal care facility, and were provided with food and water ad libitum; they were exposed to a 12:12 h light-dark cycle at room temperature. All procedures were approved by The Animal Care and Use Committee of Ewha Womans University School of Medicine, Seoul, Korea (ESM 10-0153).

Mouse model of TNBS-induced colitis. Colitis was induced by the rectal administration of $2 \mathrm{mg} / 100 \mathrm{ml}$ of TNBS (Sigma-Aldrich, St. Louis, MO, USA) in 45\% ethanol (Merck, Darmstadt, Germany) using a vinyl catheter positioned $3.5 \mathrm{~cm}$ proximal to the anus. During the procedure, the mice were anaesthetized using Rumpun/Zoletil. Following the instillation of the catheter, the animals were kept vertical for $30 \mathrm{sec}$. The control mice underwent identical procedures, but were instilled with $45 \%$ ethanol dissolved in phosphate-buffered saline (PBS). The mice were monitored daily for survival, body weight, rectal bleeding and stool consistency. All animals were sacrificed on day 5 of the experiment by cervical dislocation.

Mouse models of DSS-induced colitis. For acute DSS-induced colitis, the mice were administered 4\% DSS (molecular weight, 36,000-50,000; MP Biomedicals, Solon, OH, USA) in their drinking water for 7 days. The control animals were administered distilled water. For the induction of chronic colitis, mice were administered 2\% DSS in their drinking water for 5 days, followed by 5 days of the administration of distilled water. This cycle was repeated 3 times. The mice were monitored daily for survival, body weight, rectal bleeding and stool consistency.
Histological analysis. The large and small intestine and spleen tissues were fixed in $4 \%$ paraformaldehyde and embedded in paraffin. Fixed tissues were cut into 5-mm-thick sections, placed on glass slides and deparaffinized. The sections were stained with hematoxylin and eosin (H\&E) and observed under a light microscope.

\section{Cell preparation}

Lamina propria $(L P)$ cell isolation. Segments of the small intestine were incubated for $30 \mathrm{~min}$ at $37^{\circ} \mathrm{C}$ with fluorescence-activated cell sorting (FACS) buffer [PBS containing $10 \%$ fetal bovine serum (FBS), $20 \mathrm{mM}$ Hank's balanced salt solution, $100 \mathrm{U} / \mathrm{ml}$ penicillin, $100 \mathrm{mg} / \mathrm{ml}$ streptomycin, $1 \mathrm{mM}$ sodium pyruvate, $10 \mathrm{mM}$ ethylenediaminetetraacetic acid (EDTA) and $10 \mathrm{mg} / \mathrm{ml}$ polymyxin B] to remove epithelial cells, and were washed extensively with PBS. The segments were then digested with 400 Mandl $\mathrm{U} / \mathrm{ml}$ collagenase D and $10 \mathrm{mg} / \mathrm{ml}$ DNase I (both from Roche Diagnostics GmbH, Mannheim, Germany) in RPMI-1640/10\% FBS solution with continuous stirring at $37^{\circ} \mathrm{C}$ for $45-90 \mathrm{~min}$. EDTA was added $(10 \mathrm{mM})$, and the cell suspension was incubated for an additional $5 \mathrm{~min}$ at $37^{\circ} \mathrm{C}$. After washing, the cells were subjected to density gradient centrifugation in 40/75\% Percoll (approximate density, 1.058/1.093 g/ml), and LP leukocytes harvested from the interface were washed with PBS.

Basal membrane (BM) cell isolation. For BM isolation, mice were sacrificed by cervical dislocation and their limbs removed. The BM was flushed from the medullary cavities of femurs and tibias in RPMI-1640 medium using a 25-gauge needle. Cell suspensions were collected though a $70-\mathrm{mm}$ cell strainer and centrifuged. After the cells were pelleted, erythrocytes were removed by incubation with RBC lysis solution $\left(0.15 \mathrm{M} \mathrm{NH}_{4} \mathrm{Cl}, 10 \mathrm{mM} \mathrm{NaHCO}{ }_{3}, 10 \mathrm{mM}\right.$ EDTA-disodium in distilled water) and washed with PBS.

Peyer's patch (PP) and spleen (SP) cell isolation. Freshly isolated tissues were placed on a cell strainer ( $70 \mu \mathrm{m}$ pore size $)$ in a dish ( $6 \mathrm{~cm}$ in diameter) containing RPMI-1640 medium and $10 \%$ FBS. Tissues were mechanically disrupted using a plunger and cells were released through a cell strainer. After collection of the cells in a 15-ml conical tube, the cells were centrifuged and the erythrocytes removed using RBC lysis solution.

Flow cytometry. In order to compare the cellular profiles following the induction of colitis, cells from the BM, SP, PP and LP were resuspended in FACS buffer. The cells were subsequently incubated with the following antibodies for $30 \mathrm{~min}$ at $4^{\circ} \mathrm{C}$ (all from BioLegend, San Diego, CA, USA): fluorescein isothiocyanate (FITC)-labeled anti-mouse Ly-6G/Ly-6C (Gr-1, RB6-8C5), phycoerythrin (PE)-labeled anti-mouse CD11b (M1/70), PE-labeled anti-mouse CD4 (GK1.5), peridinin-chlorophyll-protein complex (PerCP)-labeled anti-mouse CD4 (GK1.5), FITC-labeled anti-mouse interferon (IFN)- $\gamma$ (XMG1.2), PE-labeled anti-mouse IL-9 (RM9A4), PE-labeled anti-mouse IL-17A (TC11-18H18.1) and Alexa Fluor ${ }^{\circledR} 488$-labeled anti-mouse FoxP3 (150D). The levels of labeled proteins were then analyzed on a FACSCalibur instrument (BD Biosciences, Franklin Lakes, NJ, USA).

Statistical analysis. Values are expressed as the means \pm standard error of the mean (SEM). Survival rates were estimated 
using Kaplan-Meier plots. Non-parametric Mann-Whitney tests or two-way analysis of variance were conducted to determine significance $(\mathrm{P}<0.05)$, using GraphPad Prism software (GraphPad Software Inc., San Diego, CA, USA).

\section{Results}

Induction of colitis with DSS or TNBS. In order to examine the pathogenesis of IBD, we established mouse models of colitis using TNBS or DSS. To compare different types of intestinal inflammation caused by different chemical agents or induced for different periods of time, colitis was induced as illustrated in Fig. 1. For instance, to induce acute colitis, the intrarectal administration of TNBS, combined with ethanol to increase intestinal permeability, was used to elicit intense and sustained inflammation. In the model of DSS-induced acute colitis, the mice were administered 4\% DSS in their drinking water for 7 days (days $0-6$ ).

Differences between TNBS- and DSS-induced colitis in C57BL/6 mice. As shown in Fig. 2A, both groups of TNBSand DSS-treated mice exhibited severe inflammation, manifested by shortened, thickened and erythematous colons. In macroscopic histological observations, both groups showed a massive bowel edema and disruption of epithelial cells by large ulcerations (Fig. 2B). However, changes in body weight were more prominent in TNBS-induced colitis compared with DSS-induced colitis. In particular, the majority of the TNBS-induced mice quickly developed diarrhea and showed poor coat quality and reduced mobility, followed by significant weight loss and mortality (data not shown). By contrast, mice with DSS-induced colitis showed a delay in these reactions, manifesting on day 5 from the first day of administration, while survival was $100 \%$ (Fig. 2C and D).

Pronounced differences between acute vs. chronic DSS-induced colitis. Since IBD is characterized by chronic inflammatory responses and multiple exacerbations during disease progression, a mouse model of chronic colitis is required. The mice with TNBS-induced colitis showed a higher rate of mortality, as shown in Fig. 2D; we therefore used the model of DSS-induced colitis for studying both the acute and chronic forms of the disease. While acute colitis was induced by preparing a $4 \%$ DSS solution in drinking water for 5 days, C57BL/6 mice (a highly susceptible strain) were subjected to cyclic treatment with $2 \%$ DSS for 30 days to induce chronic inflammation (Fig. 1C). Under these conditions, as shown in Fig. 3A, chronic colitis developed, accompanied by reduced colon length, similar to that observed in DSS-induced acute colitis. Moreover, continuous weight loss was observed, particularly in the 2- to 3-day interval following DSS administration (Fig. 3B). In accordance with these signs of colitis, mice further exhibited pathological characteristics at the endpoint of the experiment, i.e., they showed necrosis, ulcerations, increase in the number of goblet cells, and infiltration of neutrophils and macrophages into the colonic mucosal and submucosal layers at 30 days post-DSS treatment (Fig. 3C). The colonic mucosal structure of the crypt was broken in both the acute and chronic disease groups, with the occurrence of colonic inflammation, resulting in a clear thickening of the mucosal and muscle layers
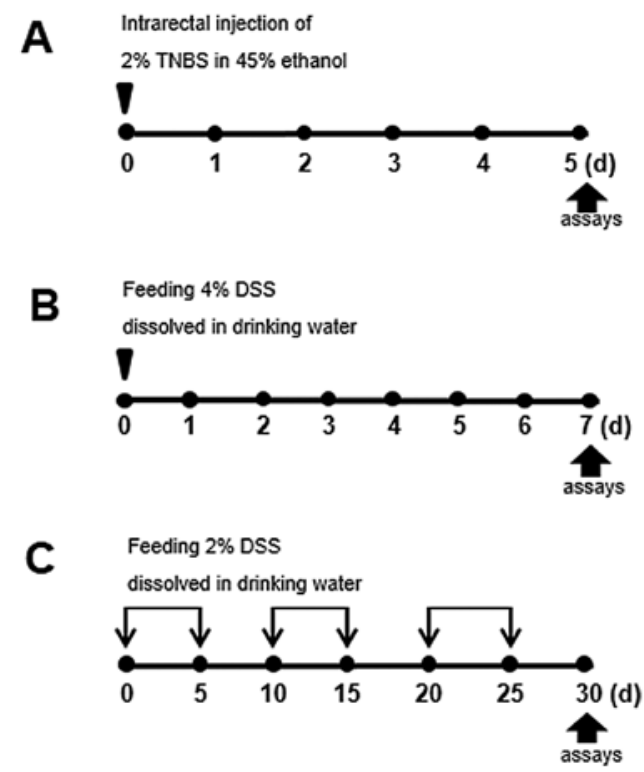

Figure 1. Experimental protocol for the induction of colitis in mice. (A) Acute colitis was induced by the rectal administration of $2 \mathrm{mg}$ 2,4,6-trinitrobenzene sulfonic acid (TNBS) in $45 \%$ ethanol or (B) by the administration $4 \%$ dextran sulfate sodium (DSS) in drinking water for 7 days. (C) Chronic colitis was induced by cyclic treatment with $2 \%$ DSS. Mice were administered $2 \%$ DSS in drinking water for 5 days and distilled water for the following 5 days. This cycle was repeated 3 times for 30 days. All mice were sacrificed on the indicated days (arrows). d, days.

in the DSS-induced colitis group as compared with the control mouse group (Figs. 2B and 3C).

Comparison of cellular profiles between acute and chronic DSS-induced colitis. We then compared acute and chronic DSS-induced colitis in terms of the mucosal innate immune response, focusing on cellular profiles. IBD is a chronic intestinal inflammatory disease characterized by the infiltration and activation of various immune cells, leading to prolonged inflammation in the intestine. In order to identify the colonic cellular populations involved in acute and chronic DSS-induced colitis, LP leukocyte cells isolated from DSS-treated mice were examined for their MDSC profile through the determination of $\mathrm{CD}_{11} \mathrm{~b}^{+} \mathrm{Gr}-\mathrm{1}^{+}$levels, as well as of the levels of Th cells, including Th1 (IFN $\gamma$-secreting, IFN $\gamma^{+}$), Th2 (IL-4-secreting, IL-4 ${ }^{+}$), Th9 (IL-9-secreting, IL-9 $\left.{ }^{+}\right)$, Th17 (IL-17-secreting, IL-17 $7^{+}$) and $\mathrm{T}_{\text {reg }}\left(\right.$ Foxp $\left.^{+}\right)$. Other lymphoid organs, including the BM, SP and PP, were also included in the determination of cellular profiles following the administration of DSS.

As shown in Fig. 4A, we observed a significant increase in the number of CD11 b $\mathrm{br}^{+} 1^{+}$cells in the SP and PP of the acute and chronic colitis mouse groups, as compared with the controls. However, the number of $\mathrm{CD} 11 \mathrm{~b}^{+} \mathrm{Gr}-1^{+}$cells decreased in the BM and increased in the LP only in the acute colitis group. As regards $\mathrm{CD} 4^{+} \mathrm{T}$ cell subsets in the LP, we observed a significantly higher percentage of IFN $\gamma^{+}$and IL- $17^{+}$cells in the mice with DSS-induced colitis in both the acute and the chronic models of colitis (Fig. 4B). Notably, the number of IL- $9^{+}$cells was markedly increased only in the chronic colitis group, whereas the number of Foxp $3^{+}$cells showed a significant decrease in both groups of mice with colitis. 
A

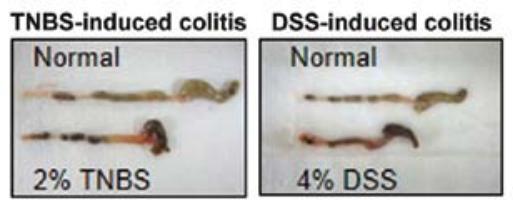

B
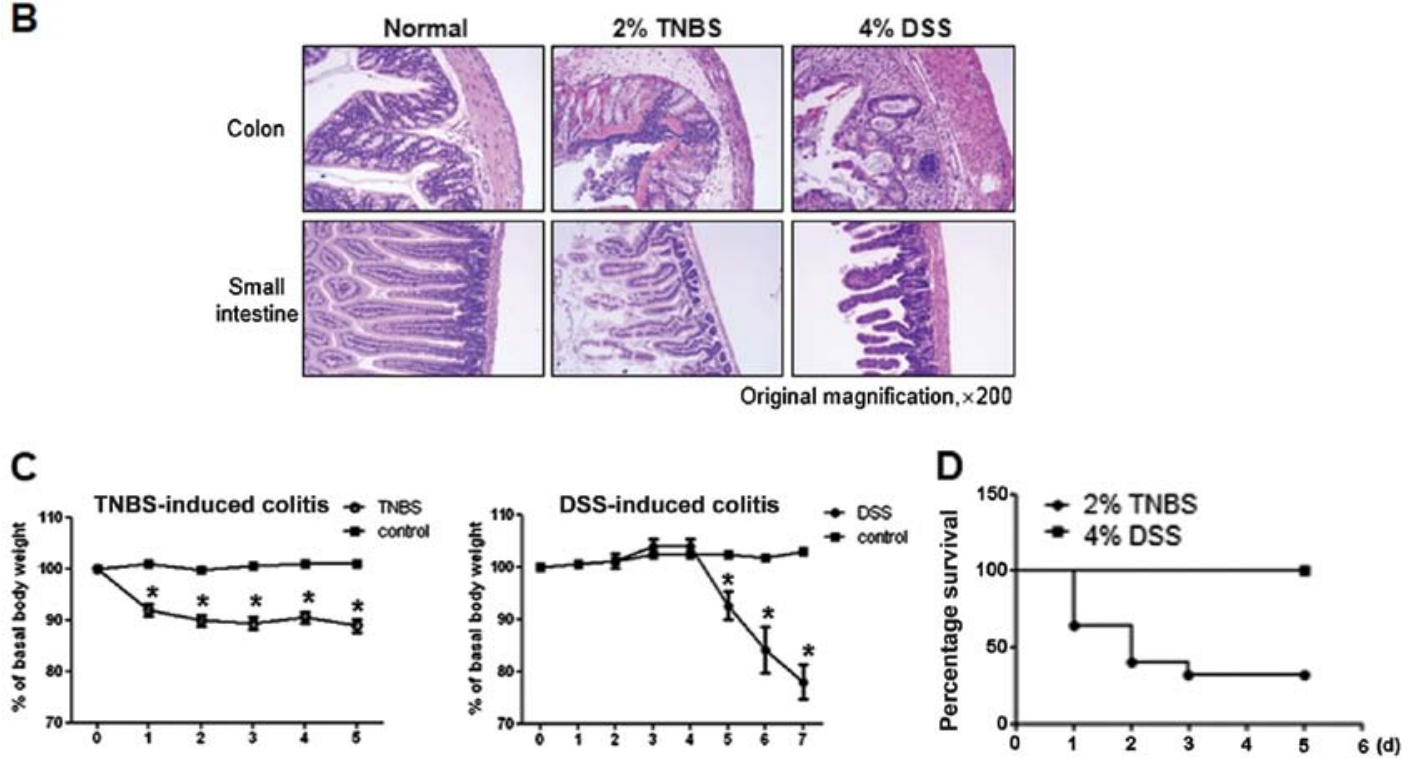

Figure 2. Induction of acute colitis. (A) Macroscopic changes in the colons of mice that received 2\% 2,4,6-trinitrobenzene sulfonic acid (TNBS) or 4\% dextran sulfate sodium (DSS). Representative image of colons from day 5 after TNBS injection (left panel) and 7 days after DSS administration (right panel). Shortening of the colon length and thickening of the colonic wall were pronounced in both TNBS- and DSS-treated mice. (B) Infiltration of inflammatory cells, mucosal necrosis, transmural inflammation, ulcerations and loss of cryptal cells observed in the colon and small intestine following induction of colitis with TNBS or DSS treatment. Tissue specimens were fixed, embedded in paraffin, sectioned and stained with hematoxylin and eosin (H\&E). (C) Daily changes in body weight during the induction of colitis. Changes in body weight percentage were calculated by dividing the body weight on the specified day by the body weight at day 0 . Data are presented as the means $\pm \mathrm{SEM}(\mathrm{n}=8)$. ${ }^{*} \mathrm{P}<0.05$ from a two-way analysis of variance. (D) Mortality rate of the mice with colitis. The survival of the mice was scored daily and is presented as a percentage; $100 \%$ denotes the survival (\%) of the mice at the beginning of the experiment. d, days.

A
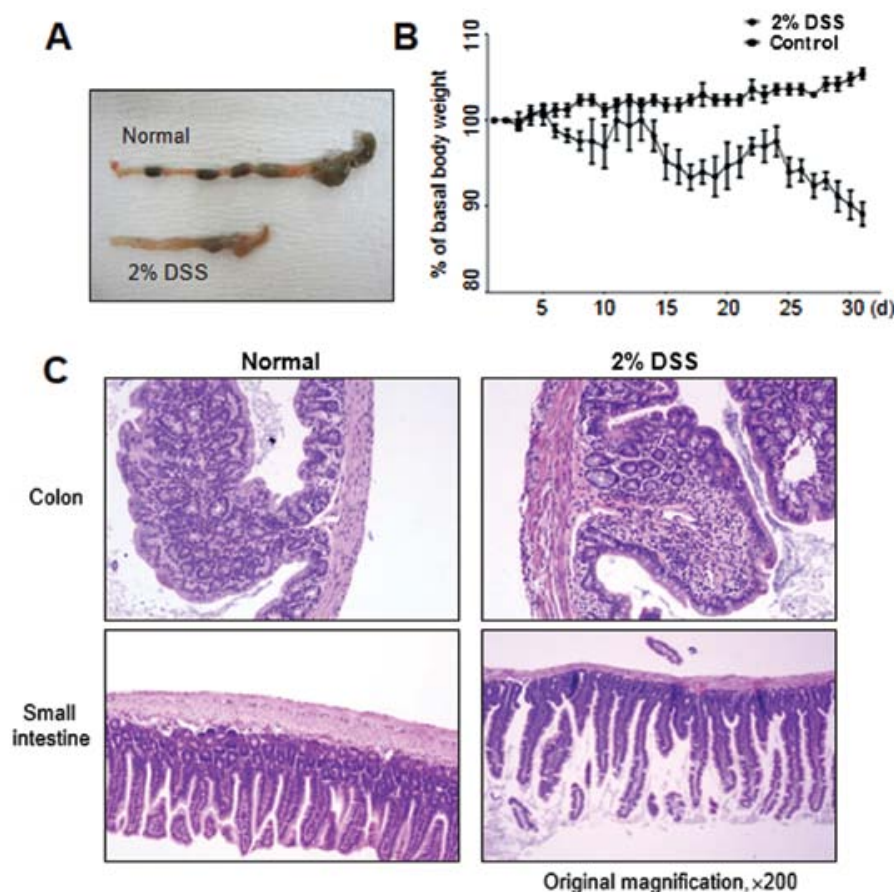

Figure 3. Induction of chronic colitis. (A) Macroscopic changes of the colon of mcie with colitis compared with healthy mice at the endpoint of the experiment; (B) body weight loss during the experiment, expressed as a percentage; and (C) histological appearance of the colon and small intestine at the endpoint of the experiment in mice with colitis compared with the healthy mice. The colon manifests mucosal ulceration, crypt damage and transmural inflammation. Chronic colitis was induced in C57BL/6 mice by 3 cycles consisting of 5 days of $2 \%$ dextran sulfate sodium (DSS) administration in drinking water followed by a 5-day interval where distilled water was administered to the mice. The third cycle was completed on day 30 . d, days. 

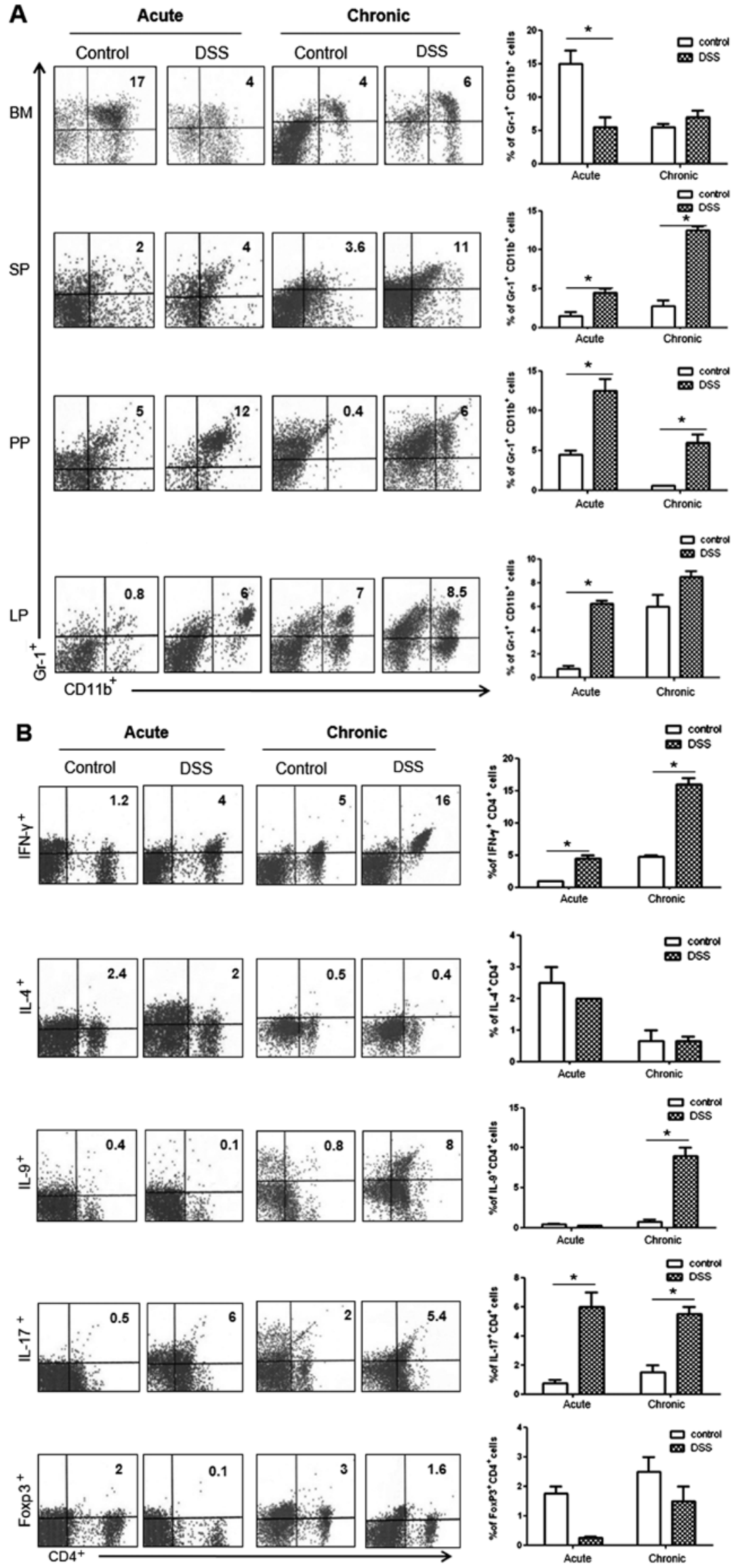

Figure 4. Cellular profiles during acute and chronic colitis induced by dextran sulfate sodium (DSS). (A) Levels of myeloid-derived suppressor cells (MDSCs) CD1 $1 \mathrm{~b}^{+} \mathrm{Gr}-1^{+}$within the basal membrane (BM), spleen (SP), Peyer's patch (PP) and lamina propria (LP) of mice with acute and chronic colitis, analyzed by flow cytometry at 7 days (acute) or 30 days (chronic) following DSS administration. (B) The proportions of CD4 $4^{+} \mathrm{T}$ cells from the LP were determined according to the intracellular levels of cytokines, for IFN $\gamma$-secreting T cells (IFN $\left.\gamma^{+}\right)$, IL-4-secreting T cells (IL-4), IL-9-secreting T cells (IL-9 $)$, Th17 cells $\left(\mathrm{IL}-17^{+}\right)$, and $\mathrm{T}_{\text {reg }}$ cells $\left(\right.$ Foxp $\left.^{+}\right)$(B). The data are expressed as the means \pm SEM ("P $<0.05$ from a non-parametric Mann-Whitney test). 
A

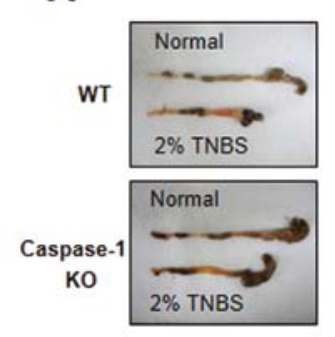

B

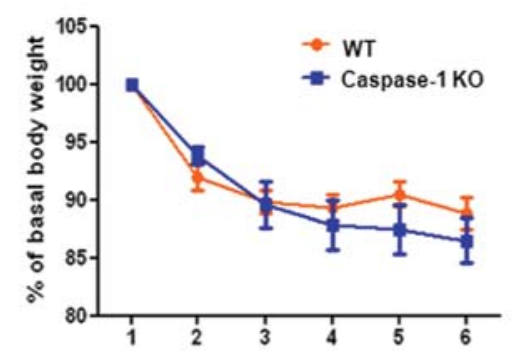

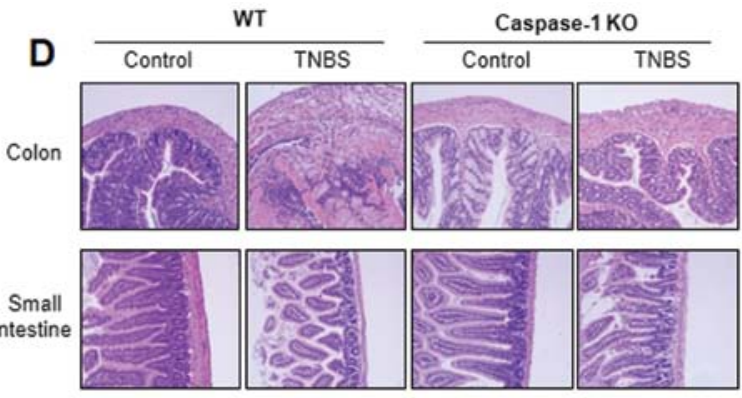

Original magnification, $\times 200$

C

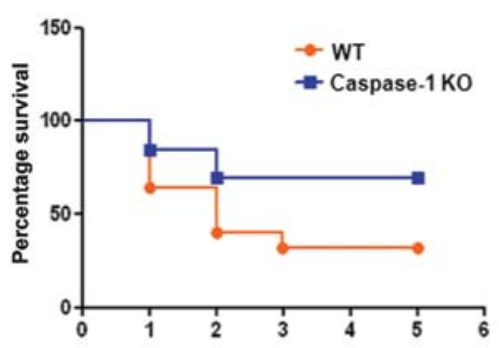

Figure 5. Induction of acute colitis in C57BL6 wild-type (WT) and caspase-1 knockout (KO) mice by the intrarectal injection of 2\% 2,4,6-trinitrobenzene sulfonic acid (TNBS), clinical and macroscopic observations. (A) Macroscopic appearance of colons harvested from TNBS-treated and control mice; (B) body weight loss; and (C) percentage of survival and (D) histological appearance of the colon and small intestine. Upon sacrifice of the control and TNBS-treated mice, paraffin-embedded tissue sections were stained with hematoxylin and eosin (H\&E). Data are presented as the means $\pm \operatorname{SEM}(n=8)$.

Pronounced differences between WT and caspase-1 KO mice in TNBS-induced colitis. IL-1 $\beta$ and IL-18 are activated by caspase-1; therefore, studies using caspase-1 KO mice are necessary to elucidate the roles of IL-1 $\beta$ and IL-18 in TNBS-induced colitis. In this study, we established acute colitis in wild-type (WT, C57BL/6) and caspase-1 KO mice by the administration of TNBS (Fig. 1A) and examined the progress of the disease. Colon shortening was more prominent in the WT mice than in the caspase-1 KO mice, and severe colitis with the thickening of the colonic wall and ulcerations were observed in TNBS-treated WT mice. By contrast, TNBS-treated caspase-1 KO mice showed smaller erosions and a mild edema in the colon (Fig. 5A). Furthermore, lower mortality rates were observed in the caspase-1 KO mice compared with the WT mice, although a similar decrease in body weight was observed in both groups (Fig. 5C). The caspase-1-dependent phenotypes were also confirmed by histological examination. Fig. 5D shows representative histological features of the colon and the small intestine in healthy WT, TNBS-treated WT and TNBS-treated caspase-1 KO mice. TNBS administration induced a marked thickening of the colonic wall, with transmural infiltration and aggregation of numerous inflammatory cells; all these features are absent in the healthy colon. Moreover, an alteration in the crypt structure and an erosion in the surface of the epithelium were clearly visible in the colons of the TNBS-treated WT mice. The TNBS-treated caspase-1 KO mice showed moderate leukocyte infiltration, a submucosal edema, and partial retention of the crypt structure and of the surface of the epithelium.

Comparison of cellular profiles between colitis in WT vs. caspase-1 KO mice. To compare the cellular profiles between WT and caspase-1 KO mice following the administration of TNBS, we performed flow cytometry. The number of
$\mathrm{CD} 11 \mathrm{~b}^{+} \mathrm{Gr}-1^{+}$MDSCs was significantly higher in the BM, SP, PP and LP of the TNBS-treated WT mice compared with the control mice. However, the TNBS-treated caspase-1 KO mice did not show a significant increase in the numbers of these cells apart from the BM cells (Fig. 6A). As regards the $\mathrm{CD}^{+}$ $\mathrm{T}$ cell subsets in the LP, the number of IL- $9^{+}$and $\mathrm{IL}-17^{+}$cells significantly increased in TNBS-treated WT mice, whereas the proportions of these cells were not increased in the TNBStreated caspase- $1 \mathrm{KO}$ mice compared with the corresponding control group. The number of Foxp $3^{+}$cells showed a significant increase only in the TNBS-treated caspase-1 KO mice (Fig. 6B).

\section{Discussion}

In this study, we compared 3 different experimental mouse models of IBD (colitis): 4\% DSS-induced (acute), 2\% DSS-induced (chronic) and 2\% TNBS-induced acute colitis, the latter studied in both WT and caspase-1 KO mice. We found that mice with chronic DSS-induced colitis showed periodic fluctuations in weight loss during the 30-day experimental period (Fig. 3B); this phenotype is more similar to the progression of IBD in humans compared with the one observed in the mouse models of acute colitis (Fig. 2C). All 3 WT mouse models of colitis showed an increase in the number of IFN- $\gamma^{+}$ and $\mathrm{IL}-17^{+} \mathrm{T}$ cells and a decrease in the number of IL- $4^{+}$ T cells. TNBS-induced colitis was less severe in the caspase-1 $\mathrm{KO}$ mice and was accompanied by an increase in the number of Foxp3 ${ }^{+}$CD4 T cells (Fig. 6).

Although the model of TNBS-induced colitis has been reported to involve $\mathrm{T}$ cell responses(3), mucosal damage from ethanol challenge is a prerequisite for establishing this model. In this study, we evaluated epithelial layer damage in terms of inflammasome activation. The final step in inflammasome acti- 
A
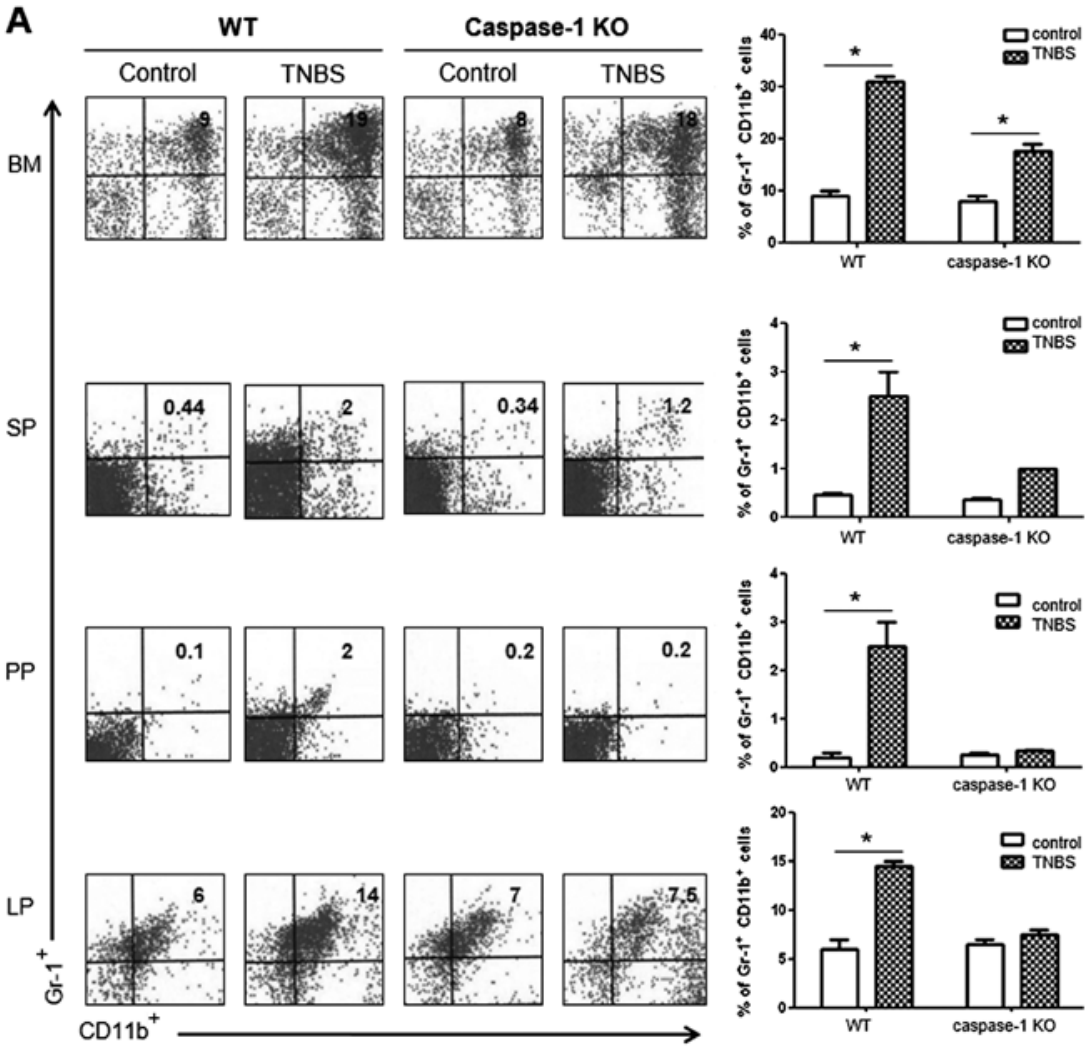

B
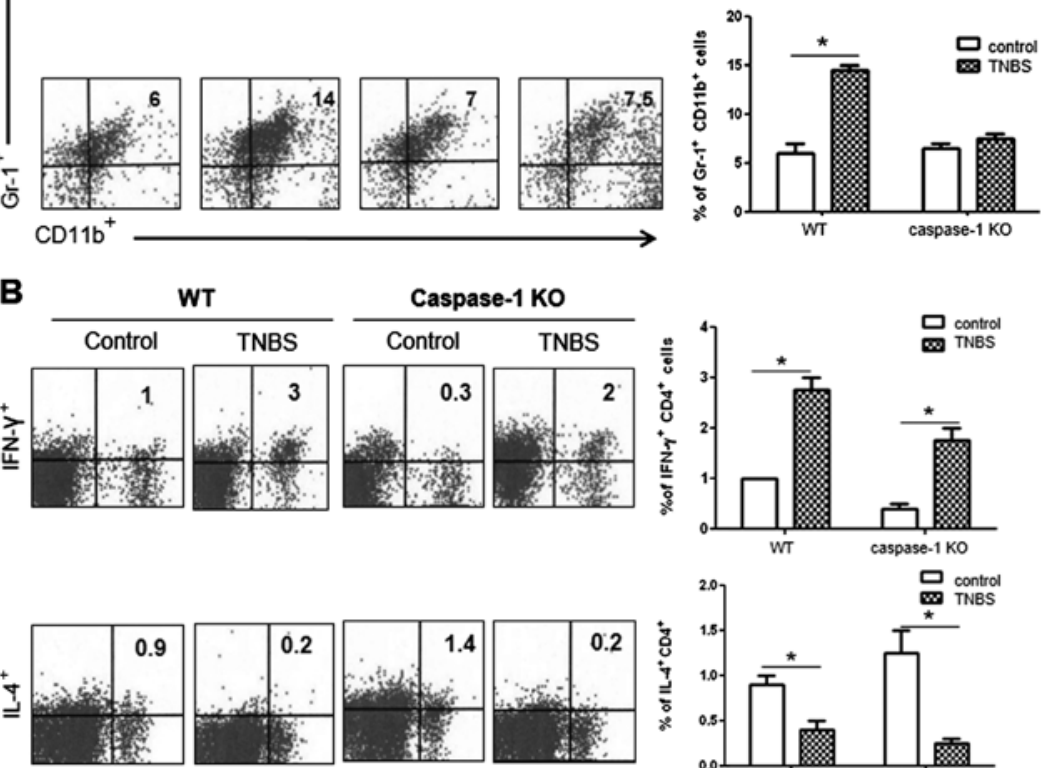

control
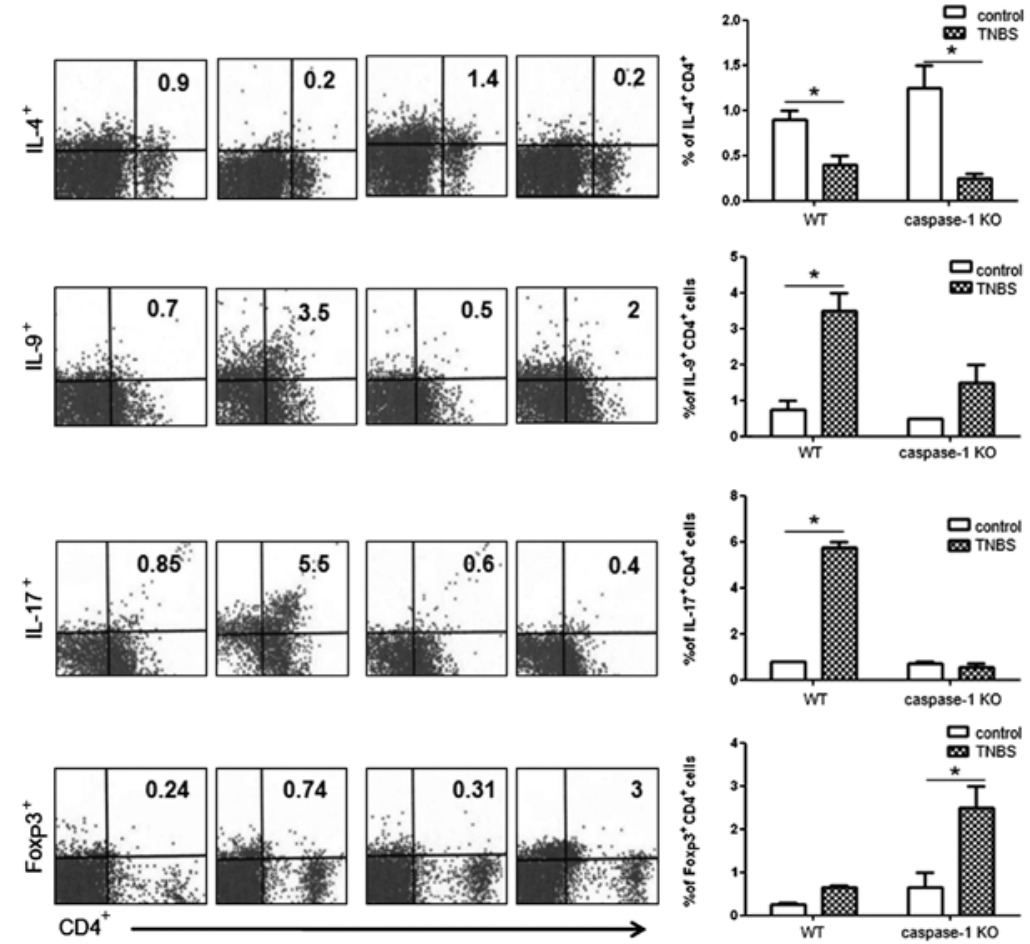

Figure 6. Caspase-1-dependent cellular profiles in acute colitis induced by 2,4,6-trinitrobenzene sulfonic acid (TNBS). (A) Levels of myeloid-derived suppressor cells (MDSCs) CD11 ${ }^{+} \mathrm{Gr}-1^{+}$within the basal membrane (BM), spleen (SP), Peyer's patch (PP) and lamina propria (LP) of wild-type (WT) and caspase-1 knockout (KO) mice, analyzed by flow cytometry 5 days following the administration of TNBS. (B) The proportions of CD4 ${ }^{+} \mathrm{T}$ cells from the LP were determined according to the intracellular levels of cytokines, for IFN $\gamma$-secreting T cells (IFN $\left.\gamma^{+}\right)$, IL-4-secreting T cells (IL-4 ${ }^{+}$), IL-9-secreting T cells (IL- ${ }^{+}$), Th17 cells $\left(\mathrm{IL}-17^{+}\right)$and $\mathrm{T}_{\text {reg }}$ cells $\left(\right.$ Foxp $\left.3^{+}\right)(\mathrm{B})$. The data are expressed as the means \pm SEM $\left({ }^{*} \mathrm{P}<0.05\right.$ from a non-parametric Mann-Whitney test). 
vation involves caspase-1 activation, leading to the cleavage of pro-IL-1 $\beta$ to the active form of IL-1 $\beta$. We challenged caspase-1 KO mice with TNBS to induce colitis, and found that acute TNBS-induced colitis was accompanied by an increase in the number of IFN $\gamma^{+}$and Foxp3 $3^{+}$cells but not in the number of IL- $17^{+}$cells. Considering that IL-1 $\beta$ enhances Th17 (IL-17 $\left.{ }^{+}\right)$ cell differentiation, the observed decrease in the number of IFN $\gamma^{+}$cells upon TNBS challenge in the caspase- $1 \mathrm{KO}$ mice was expected (Fig. 6B).

It was previously reported that NLRP3 KO mice are protected against DSS- and TNBS-induced colitis, possibly due to an increase in the number of tolerogenic $\mathrm{CD} 103^{+}$dendritic cells (11). In the present study, we focused on the immune-suppressive cell population of MDSCs. MDSCs are poorly characterized in human and mouse models of IBD; there are only limited studies available on MDSCs in colitis. It has been previously reported that the transplantation of $\mathrm{CD} 11 \mathrm{~b}^{+} \mathrm{Gr}-1^{+}$splenic cells into mice with DSS-induced colitis improved disease parameters (16) and suppressed the development of CD8-induced autoimmune enterocolitis (17). By contrast, there are several reports on the pro-inflammatory role of myeloid cells in models of IBD. For example, during chronic colitis, adoptively transferred Ly $6 C^{\text {high }}$ monocytes are recruited to the colon and differentiate into inflammatory DCs and macrophages (18), while neutrophils $\left(\mathrm{CD} 11 \mathrm{~b}^{+} \mathrm{Ly}_{6 \mathrm{G}}{ }^{+} \mathrm{Ly} 6 \mathrm{C}^{\mathrm{int}}\right)$ act as antigen-presenting cells to trigger $\mathrm{T}$ cell proliferation (19). These results suggest that MDSCs can provide activation signals for effector $\mathrm{T}$ cells in the LP of the colon. In our study, since severe inflammatory changes occured in the DSS- and TNBS-challenged mice and

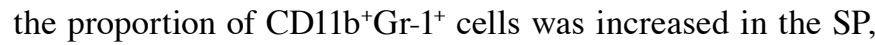
PP and LP, MDSCs may contribute to the pro-inflammatory response. However, the increase in the number of MDSCs that we observed may also be due to the modulation of immune responses. The most notable difference between the caspase-1 $\mathrm{KO}$ and WT mice was that no significant increase was observed

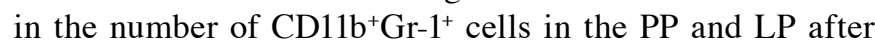
TNBS challenge in the caspase-1 KO mice (Fig. 6A). It has been reported that IL-1 $\beta$ expression correlates with the early recruitment of MDSCs to inflammatory tissue (20), and this result could explain the decrease in the number of MDSCs in the caspase- $1 \mathrm{KO}$ mice, where IL-1 $\beta$ expression is expected to

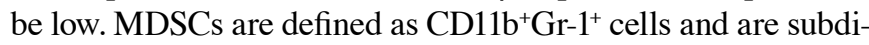
vided into granulocytic $\left(\mathrm{CD} 11 \mathrm{~b}^{+} \mathrm{Ly} 6 \mathrm{G}^{+} \mathrm{Ly} 6 \mathrm{C}^{\mathrm{int}}\right)$ and monocytic $\left(\right.$ CD11b $^{+}$Ly6G-Ly6C $\left.{ }^{+ \text {high }}\right)$. As we used anti-mouse Ly-6G/Ly-6C (Gr-1, RB6-8C5) antibody binding to both Ly6G and Ly6C, we could not differentiate granulocytic from monocytic MDSCs in our study and thus could not compare these populations of cells.

In conclusion, among $\mathrm{CD}^{+}{ }^{+} \mathrm{T}$ cell subsets in the $\mathrm{LP}$, the number of $\mathrm{IFN} \gamma^{+}$and $\mathrm{IL}-17^{+}$, but not that of $\mathrm{IL}-4^{+}$cells, increased in the 3 different mouse models of IBD. The discrepancies in histopathological changes between the WT and caspase- $1 \mathrm{KO}$ mice further suggest that IBD pathogenesis may be associated with the caspase-1-mediated inflammasome pathway in TNBS-induced colitis, and is accompanied by an increase in the number of $\mathrm{T}_{\text {reg }}\left(\mathrm{Foxp}^{+}\right)$cells.

\section{Acknowledgements}

This study was partly supported by the Basic Science Research Program through the National Research Foundation of Korea (NRF) funded by the Ministry of Science, ICT and Future Planning (2013R1A1A3A04006995 to S.-Y.W.) and by the Korean Government (MRC-2010-0029353 to J.L.K).

\section{References}

1. Morris GP, Beck PL, Herridge MS, Depew WT, Szewczuk MR and Wallace JL: Hapten-induced model of chronic inflammation and ulceration in the rat colon. Gastroenterology 96: 795-803, 1989.

2. Wirtz S, Neufert C, Weigmann B and Neurath MF: Chemically induced mouse models of intestinal inflammation. Nat Protoc 2: 541-546, 2007.

3. Neurath MF, Fuss I, Kelsall BL, Stuber E and Strober W: Antibodies to interleukin 12 abrogate established experimental colitis in mice. J Exp Med 182: 1281-1290, 1995.

4. Elson CO, Beagley KW, Sharmanov AT, et al: Hapten-induced model of murine inflammatory bowel disease: mucosa immune responses and protection by tolerance. J Immunol 157: 2174-2185, 1996.

5. Okayasu I, Hatakeyama S, Yamada M, Ohkusa T, Inagaki Y and Nakaya R: A novel method in the induction of reliable experimental acute and chronic ulcerative colitis in mice. Gastroenterology 98: 694-702, 1990.

6. Dieleman LA, Ridwan BU, Tennyson GS, Beagley KW, Bucy RP and Elson CO: Dextran sulfate sodium-induced colitis occurs in severe combined immunodeficient mice. Gastroenterology 107: 1643-1652, 1994.

7. Yamada M, Ohkusa T and Okayasu I: Occurrence of dysplasia and adenocarcinoma after experimental chronic ulcerative colitis in hamsters induced by dextran sulphate sodium. Gut 33: 1521-1527, 1992.

8. Ishiguro Y: Mucosal proinflammatory cytokine production correlates with endoscopic activity of ulcerative colitis. J Gastroenterol 34: 66-74, 1999.

9. Monteleone G, Trapasso F, Parrello T, et al: Bioactive IL-18 expression is up-regulated in Crohn's disease. J Immunol 163: 143-147, 1999.

10. Kwon KH, Murakami A, Hayashi $\mathrm{R}$ and Ohigashi $\mathrm{H}$ : Interleukin-lbeta targets interleukin-6 in progressing dextran sulfate sodium-induced experimental colitis. Biochem Biophys Res Commun 337: 647-654, 2005.

11. Bauer C, Duewell P, Mayer C, et al: Colitis induced in mice with dextran sulfate sodium (DSS) is mediated by the NLRP3 inflammasome. Gut 59: 1192-1199, 2010.

12. Elinav E, Strowig T, Kau AL, et al: NLRP6 inflammasome regulates colonic microbial ecology and risk for colitis. Cell 145: 745-757, 2011.

13. Nam JH: Regulation of obesity and non-alcoholic fatty liver disease by modulation of the gut micobiota through inflammasome; its mechanism and potential for clinical use. J Bacteriol Virol 42: 359-362, 2012.

14. Siegmund B, Lehr HA, Fantuzzi G and Dinarello CA: IL-1beta-converting enzyme (caspase-1) in intestinal inflammation. Proc Natl Acad Sci USA 98: 13249-13254, 2001.

15. Gabrilovich DI and Nagaraj S: Myeloid-derived suppressor cells as regulators of the immune system. Nat Rev Immunol 9: $162-174,2009$.

16. Zhang R, Ito S, Nishio N, Cheng Z, Suzuki H and Isobe KI: Dextran sulphate sodium increases splenic Grl(+)CD11b(+) cells which accelerate recovery from colitis following intravenous transplantation. Clin Exp Immunol 164: 417-427, 2011.

17. Haile LA, von Wasielewski R, Gamrekelashvili J, et al: Myeloidderived suppressor cells in inflammatory bowel disease: a new immunoregulatory pathway. Gastroenterology 135: 871-881, 2008.

18. Rivollier A, He J, Kole A, Valatas V and Kelsall BL: Inflammation switches the differentiation program of Ly6Chi monocytes from antiinflammatory macrophages to inflammatory dendritic cells in the colon. J Exp Med 209: 139-155, 2012.

19. Ostanin DV, Kurmaeva E, Furr K, et al: Acquisition of antigenpresenting functions by neutrophils isolated from mice with chronic colitis. J Immunol 188: 1491-1502, 2012.

20. Tu S, Bhagat G, Cui G, et al: Overexpression of interleukin-1beta induces gastric inflammation and cancer and mobilizes myeloid-derived suppressor cells in mice. Cancer Cell 14: 408-419, 2008. 